Bryn Mawr College

Scholarship, Research, and Creative Work at Bryn Mawr

College

2006

\title{
Magnetic Properties of One-Dimensional Quasiperiodic Co/Pt Multilayers
}

L. Y.Zhu

Xuemei Cheng

Bryn Mawr College, xcheng@brynmawr.edu

C. L. Chien

Let us know how access to this document benefits you.

Follow this and additional works at: http://repository.brynmawr.edu/physics_pubs

Part of the Physics Commons

\section{Custom Citation}

L. Y. Zhu, X. M. Cheng and C. L. Chien, J. Appl. Phys. 99, 8C902 (2006).

This paper is posted at Scholarship, Research, and Creative Work at Bryn Mawr College. http://repository.brynmawr.edu/physics_pubs/59

For more information, please contact repository@brynmawr.edu. 


\title{
Magnetic properties of one-dimensional quasiperiodic Co/Pt multilayers
}

\author{
L. Y. Zhu, ${ }^{\text {a) }}$ X. M. Cheng, and C. L. Chien \\ Department of Physics and Astronomy, Johns Hopkins University, Baltimore, Maryland 21218
}

(Presented on 1 November 2005; published online 20 April 2006)

\begin{abstract}
We have fabricated Fibonacci $[\mathrm{Co} / \mathrm{Pt}]$ multilayers with two constituent $\mathrm{Co} / \mathrm{Pt}$ bilayers, as well as periodic multilayers of the same $\mathrm{Co} / \mathrm{Pt}$ bilayers, all of which exhibit perpendicular magnetic anisotropy. Magnetic properties of the quasiperiodic and periodic $[\mathrm{Co} / \mathrm{Pt}]$ multilayers have been studied macroscopically by vibrating sample magnetometry and microscopically by magnetic force microscopy. The Fibonacci $[\mathrm{Co} / \mathrm{Pt}]$ multilayers show enhanced squareness in magnetic hysteresis loops compared to the corresponding periodic $[\mathrm{Co} / \mathrm{Pt}]_{n}$ multilayers, due to a smaller density of isolated nucleation sites in the saturation state. (C) 2006 American Institute of Physics.
\end{abstract}

[DOI: $10.1063 / 1.2166603$ ]

\section{INTRODUCTION}

$[\mathrm{Co} / \mathrm{Pt}]_{n}$ multilayers have been extensively studied because of their unusual attributes of perpendicular magnetic anisotropy (PMA) and potential applications in high-density perpendicular magnetic recording. ${ }^{1-5}$ The PMA originates mainly from the interfacial magnetic anisotropy between the adjacent $\mathrm{Co}$ and Pt layers. To date, most studies have been focused on periodic $[\mathrm{Co} / \mathrm{Pt}]_{n}$ multilayers, the magnetic properties of which depend on the constituent $\mathrm{Co} / \mathrm{Pt}$ bilayers as well as on the number of repeats $n^{2}{ }^{2}$ Quasiperiodic layer structures, such as those with one-dimensional (1D) Fibonacci sequence, ${ }^{6}$ are interesting variations of multilayers, as have been explored using semiconductor and optical materials. ${ }^{7,8}$ Studies of the magnetic Fibonacci sequences have also been reported in rare-earth $\mathrm{Gd}-\mathrm{Y}$ multilayers, where the interlayer coupling strength can be tuned by the thickness of the intervening Y layers. ${ }^{9}$ However, quasiperiodic $[\mathrm{Co} / \mathrm{Pt}]$ multilayers have not been reported.

In this work, we have fabricated and studied quasiperiodic $[\mathrm{Co} / \mathrm{Pt}]$ multilayers with a Fibonacci sequence using two building blocks of $A=[\operatorname{Co}(6 \AA) / \operatorname{Pt}(6 \AA)]$ and $B$ $=[\mathrm{Co}(6 \AA) / \operatorname{Pt}(13 \AA)]$. The magnetic properties of the Fibonacci sample as revealed macroscopically by vibrating sample magnetometry (VSM) and microscopically by magnetic force microscopy (MFM) imaging are different from those of the periodic $A_{8}$ and $B_{8}$ multilayers of the constituent building blocks.

\section{EXPERIMENT}

The quasiperiodic and periodic $[\mathrm{Co} / \mathrm{Pt}]$ multilayers using $A=[\operatorname{Co}(6 \AA) / \operatorname{Pt}(6 \AA)]$ and $B=[\operatorname{Co}(6 \AA) / \operatorname{Pt}(13 \AA)]$, grown on a $100-\AA$ Pt buffer layer and capped with a $20-\AA$ Pt, have been made by dc magnetron sputtering with a base pressure of $10^{-8}$ Torr. In all of these multilayers, the [Co $(6$ $\AA)]$ remains unchanged, except using either [Pt (6 ̊) ] or [Pt $(13 \AA)]$. The quasiperiodic Fibonacci and periodic structures were examined by small-angle $\mathrm{x}$-ray reflectivity measured on a four-circle X-ray diffractometer (Philips X'Pert-MRD) with $\mathrm{Cu} K_{\alpha}(\lambda=1.5405 \AA)$ radiation. The macroscopic magnetic

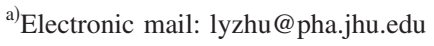

properties were measured by a DMS model 10 vector VSM with the magnetic field applied perpendicular to the film plane. MFM (Veeco extended multimode MFM) imaging was performed with or without an external field perpendicular to the film plane provided by a pair of Helmholtz coils. The samples for the MFM imaging were premagnetized by an electromagnet. A proper lift scan height was chosen during the MFM measurements to avoid changing the magnetic patterns of the sample by the MFM tip.

\section{RESULTS AND DISCUSSION}

A Fibonacci sequence consists of two building blocks, $A$ and $B$, whose order follows a specific substitution rule. The generation of a Fibonacci sequence is defined according to the number of times the substitution rule has been applied. The first generation is just $A$. In the higher generations, one substitutes block $A$ of the previous generation with $A B$ and substitutes block $B$ of the previous generation with $A$. For example, the structures of the first five generations are $A, A B, A B A, A B A A B$, and $A B A A B A B A$. The structure of the fifth generation Fibonacci sequence $(A B A A B A B A)$ is shown schematically in Fig. 1(a). The thickness ratio $d_{B} / d_{A}$, where $d_{A}$ and $d_{B}$ are the thicknesses of the building blocks $A$ and $B$, respectively, has been designed to be the golden mean ratio $\tau=(\sqrt{5}+1) / 2=1.6$.

The small-angle $\mathrm{x}$-ray diffraction (XRD) pattern for a periodic $[\mathrm{Co} / \mathrm{Pt}]_{n}$ multilayer consists of a series of equally spaced peaks. On the other hand, the small-angle XRD pattern for the Fibonacci $[\mathrm{Co} / \mathrm{Pt}]$ samples such as the tenth generation [Fig. 1(b)] can be divided into two groups: thickness peaks and Fibonacci peaks. The peaks labeled with $A, B$, and $A+B$ correspond to the thicknesses of the building blocks $A$ and $B$ and to the combined thickness of $A+B$. The determined thicknesses of $d_{A}(12.0 \AA)$ and $d_{B}(20.0 \AA)$ are in good agreement with our designed values. The seven peaks indexed by $(n, m)$ are Fibonacci peaks from the Fourier transform spectrum of a Fibonacci multilayer, consisting of the peaks at the scattering vector $k=2 \pi d^{-1}(n+m \tau)$, where $n$ and $m$ are integers and $d=\tau d_{A}+d_{B}$ is the average lattice parameter. ${ }^{10}$ The calculated $k$ from the designed structure parameters and the experimentally determined $k$ according to the formula $k=4 \pi \lambda^{-1} \sin \theta$ shown in Fig. 1(c) are in good 
(a)

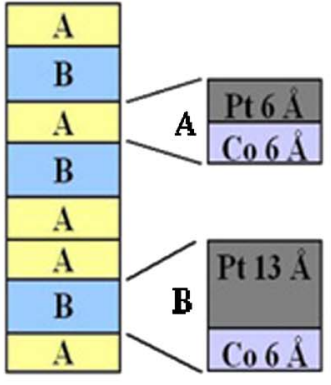

(c)

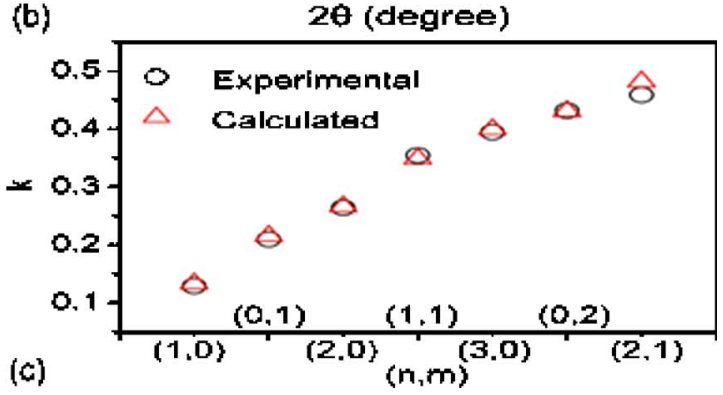

FIG. 1. (Color online) (a) Schematics of a fifth generation Fibonacci multilayer $(A B A A B A B A)$ with two building blocks $A$ and $B$. (b) X-ray diffraction pattern of the tenth generation Fibonacci sequence; $(n, m)$ labels the corresponding Fibonacci peaks. (c) Comparison between experimental values of the scattering vector $k$ $=4 \pi \lambda^{-1} \sin \theta$ (open circles) and calculated values derived from $k=2 \pi d^{-1}(n$ $+m \tau)$ (open up triangles) for each pair of $(n, m)$. agreement. The few unlabeled and weaker peaks in Fig. 1(b) are probably due to the thicknesses of different subunits consisting of several adjacent layers. The XRD results demonstrate that the Fibonacci $[\mathrm{Co} / \mathrm{Pt}]$ multilayer indeed has the Fibonacci sequence structure as designed.

The field $(H)$ dependence of the magnetization $(M)$ for a fifth generation Fibonacci sample of $A B A A B A B A$ is shown in Fig. 2. The $M-H$ loops of the two corresponding periodic samples $A_{8}=[\operatorname{Co}(6 \AA) / \operatorname{Pt}(6 \AA)]_{8}$ and $B_{8}=[\operatorname{Co}(6 \AA) /$ $\operatorname{Pt}(13 \AA)]_{8}$, grown in the same batch with the Fibonacci sample, are also shown in Fig. 2. All three samples show perpendicular magnetic anisotropy. We use the squareness ratio $S=M_{r} / M_{s}$ to quantitatively describe the sharpness of the hysteresis loop, where $M_{r}$ is the remnant magnetization and $M_{s}$ is the saturated magnetization. The sample $A_{8}$ with $S=0.68$ has the smallest remanence and the most slanted hysteresis loop with a gradual magnetization reversal process. The sample $B_{8}$ with $S=0.90$ has a larger remanence and sharper magnetization reversal. Compared with $A_{8}$ and $B_{8}$, the Fibonacci sample has the largest squareness ratio $(S$ $=0.98)$ and the sharpest magnetization reversal. One notes that the averaged Pt thickness for Fibonacci sample is $8.6 \AA$, in between those of $A_{8}$ and $B_{8}$.

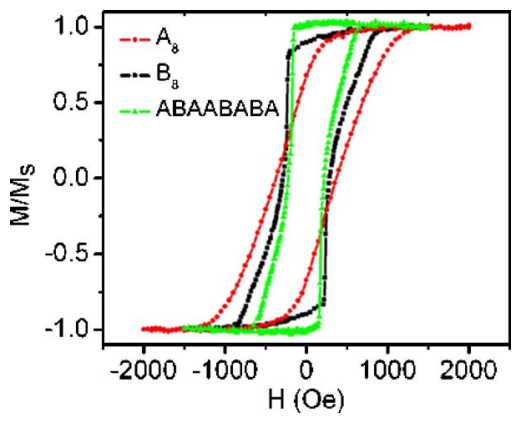

FIG. 2. (Color online) Room-temperature hysteresis loops of $[\mathrm{Co}(6 \AA) / \operatorname{Pt}(6 \AA)]_{8} \quad\left(\mathrm{~A}_{8}\right.$, solid circles $),[\mathrm{Co}(6 \AA) / \operatorname{Pt}(13 \AA)]_{8} \quad\left(B_{8}\right.$, solid squares), and fifth generation Fibonacci ( $A B A A B A B A$, solid up triangles) samples.
In order to understand the differences in the macroscopic magnetization reversal behavior among $A_{8}, B_{8}$, and the Fibonacci sample, MFM imaging was performed to reveal the microscopic domain patterns at the remnant state $(H=0)$ of each sample after being saturated by a field of $3 \mathrm{kOe}$ applied perpendicular to the film plane. At the remnant state, there are few isolated dark ("down") bubble domains in all three samples but with different densities. Previously, transmission $\mathrm{x}$-ray microscopy in $[\mathrm{Co} / \mathrm{Pt}]_{n}$ multilayers shows that before the avalanche occurs, the number of domains is the same as that of the nucleation sites at the apparent saturation state. ${ }^{3}$ In our case, the number of nucleation sites at the apparent saturation state can therefore be measured from the number of domains at the remnant state. The Fibonacci sample with the sharpest magnetization reversal reveals very few nucleation sites, as compared to the other two samples with more gradual magnetization reversal. The MFM measurements were repeated in different regions of the samples to obtain an averaged number of nucleated bubble domains within the $50 \times 50 \mu \mathrm{m}^{2}$ imaging area. The average values are 6 for the sample $A B A A B A B A(S=0.98), 28$ for sample $B_{8}(S=0.90)$, and 300 for sample $A_{8}(S=0.68)$. This clearly shows that the squareness ratio $S$ increases for samples with a fewer average number of nucleated bubble domains. Therefore, there is a strong correlation between the number of nucleation sites and the subsequent magnetization reversal process. In particular, the Fibonacci sample has the fewest number of the nucleation sites and the sharpest reversal process.

Since the interface and surface quality is critical for the formation of nucleation sites, the surface roughness for periodic and quasiperiodic $[\mathrm{Co} / \mathrm{Pt}]$ multilayers was measured by atomic force microscopy (AFM). The AFM results show the same small surface roughness of 1-3 A measured in both the periodic and Fibonacci $[\mathrm{Co} / \mathrm{Pt}]$ multilayers. The smaller number of the nucleation sites in the Fibonacci sample is apparently unrelated to the surface roughness.

To explore the role of the nucleation sites during the magnetization reversal process, following several distinctive 


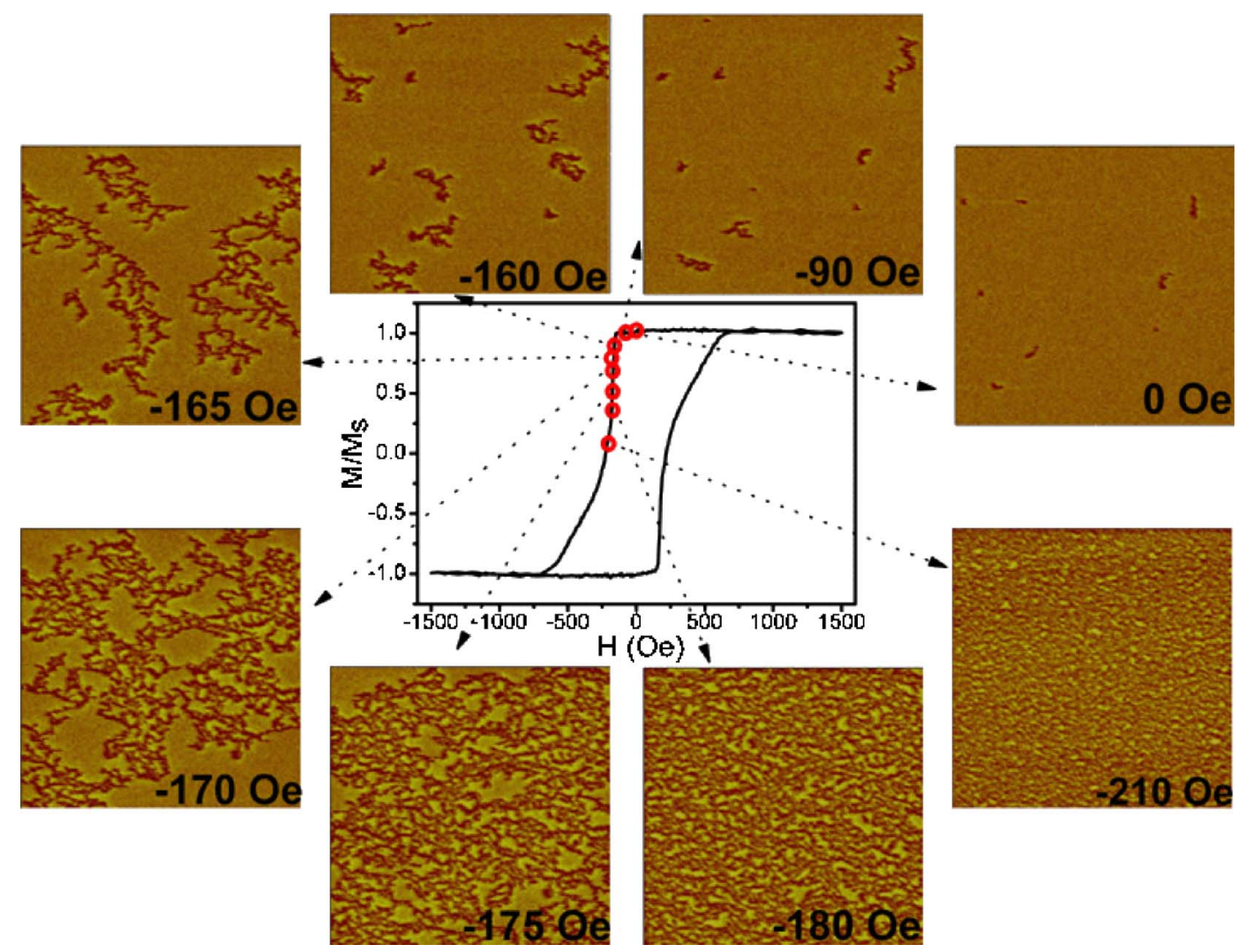

FIG. 3. (Color online) Hysteresis loop of $A B A A B A B A$ Fibonacci $[\mathrm{Co} / \mathrm{Pt}]$ multilayer. MFM images at several field values on the hysteresis loop are also shown. Approximate external fields are marked on the hysteresis loop and connected by arrows for each image.

features of the hysteresis loop, a series of representative MFM images, over the same $50 \times 50 \mu \mathrm{m}^{2}$ area on the Fibonacci $A B A A B A B A$ sample, was taken as the magnetic field was reduced from zero to more negative values as shown in Fig. 3. Initially, at the remnant state $(0$ Oe), the sample has few reversed domains located at isolated nucleation sites. With increasing the magnitude of the reversal field from 0 to -90 Oe, even though the magnetization is very close to saturation, these isolated bubble domains grow in size. The second distinctive feature of the hysteresis loop is the precipitously sharp reduction of magnetization near $-170 \mathrm{Oe}$, at which a sudden avalanche of labyrinth stripe domains was observed. These labyrinth stripes propagate through the whole sample very rapidly from -170 to -180 Oe. The third feature of the hysteresis loop is the much lower reduction of the magnetization. In this region, the MFM shows mainly a change in the width of "up" versus "down" domains. The ratio of the bright area versus dark area agrees well with the macroscopic magnetization.

The magnetization reversal in multilayers with perpendicular magnetic anisotropy has been attributed to two main reversal mechanisms: domain wall nucleation and domain wall motion. ${ }^{11}$ For $[\mathrm{Co} / \mathrm{Pt}]_{n}$ multilayers, the nucleation mode dominates in the early magnetization reversal stage, as the case for the Fibonacci sample shown by direct domain imaging. A recent report using transmission x-ray microscopy shows the existence of a large number of nucleation sites in the saturation state. ${ }^{3}$ The bubble domains nucleate from these sites. As the onset of reversal is approached, the size of the reversed domains increases by incorporating new nucleation sites in the adjacent areas. These aspects are also observed in the Fibonacci sample but from the remnant state onward.

\section{CONCLUSIONS}

In conclusion, we have successfully fabricated quasiperiodic Fibonacci $[\mathrm{Co} / \mathrm{Pt}]$, as well as periodic multilayers of the same constituent layers, by dc magnetron sputtering. Compared to the corresponding periodic multilayers, the Fibonacci multilayers exhibit enhanced perpendicular magnetic anisotropy with a sharper hysteresis loop. MFM imaging shows a smaller density of the isolated nucleation sites in the Fibonacci sample than that in the periodic multilayers.

\section{ACKNOWLEDGMENT}

This work is supported by NSF Grant Nos. DMR 0403849 and DMR05-20491.

${ }^{1}$ D. Weller et al., J. Appl. Phys. 89, 7525 (2001).

${ }^{2}$ J. W. Knepper and F. Y. Yang, Phys. Rev. B 71, 224403 (2005).

${ }^{3}$ J. E. Davies et al., Phys. Rev. B 70, 224434 (2004).

${ }^{4}$ X. M. Cheng et al., Phys. Rev. Lett. 94, 017203 (2005).

${ }^{5}$ C. L. Canedy, X. W. Li, and G. Xiao, Phys. Rev. B 62, 508 (2000)

${ }^{6} \mathrm{R}$. Merlin et al., Phys. Rev. Lett. 55, 1768 (1985).

${ }^{7}$ P. Hawrylak and J. J. Quinn, Phys. Rev. Lett. 57, 380 (1986).

${ }^{8}$ S. N. Zhu, Y. Y. Zhu, and N. B. Ming, Science 278, 843 (1997).

${ }^{9}$ C. F. Majkrzak et al., Adv. Phys. 40, 99 (1991).

${ }^{10}$ D. Levine and P. J. Steinhardt, Phys. Rev. Lett. 53, 2477 (1984).

${ }^{11}$ V. Grolier et al., Phys. Rev. Lett. 71, 3023 (1993). 